
\title{
25 Research Square \\ Liver Failure in Pregnancy: A Retrospective Study of 62 Cases
}

\section{Zhenyan Han}

Third Affiliated Hospital of Sun Yat-sen University

\section{Jin Zhou}

Third Affiliated Hospital of Sun Yat-sen University

\section{Peizhen Zhang}

Third Affiliated Hospital of Sun Yat-sen University

\section{Zhangmin Tan}

Third Affiliated Hospital of Sun Yat-sen University

\section{Tiantian He}

Third Affiliated Hospital of Sun Yat-sen University

\section{Hongying Hou}

Third Affiliated Hospital of Sun Yat-sen University

\section{Yuzhu Yin}

Third Affiliated Hospital of Sun Yat-sen University

\section{Yuan Zhang ( $\nabla$ zylion110@163.com )}

Third Affiliated Hospital of Sun Yat-sen University

\section{Research Article}

Keywords: liver failure in pregnancy, in-hospital death, clinical characteristics, change tendency

Posted Date: May 7th, 2021

DOl: https://doi.org/10.21203/rs.3.rs-461738/v1

License: (c) (1) This work is licensed under a Creative Commons Attribution 4.0 International License. Read Full License 


\section{Abstract}

Background: Liver failure in pregnancy is a rare but potentially severe disease with a high rate of shortterm morbidity and mortality, while there is still a lack of accurate diagnosis, effective treatments and prognostic indicators for liver failure in pregnancy. This study aims to retrospectively investigate the clinical characteristics of liver failure in pregnancy caused by AFLP and hepatitis B, and to explore the potential prognostic indicators.

Methods: Sixty-two pregnant women with symptoms and signs of hepatic dysfunction, admitting to the Third Affiliated Hospital of Sun Yat-sen University between January 1, 2010 and December 31, 2019 were retrospectively recruited. The baseline clinical characteristics, in-hospital mortality and changes of important laboratory examination parameters during hospitalization were determined.

Results: The in-hospital mortality rate of liver failure in pregnancy was $27.4 \%$ and most of the deaths were recorded in the first 7 days after admission. Patients suffered in-hospital death had a significant lower gestational age, a higher incidence rate of hepatorenal syndrome and were more likely to receive hysterectomy but less likely to receive intrauterine balloon tamponade. The baseline aspartate aminotransferase, total bilirubin, indirect bilirubin and platelet levels were significantly higher, while cholinesterase, prothrombin activity and creatinine levels were significantly lower in patients with inhospital death than that in patients discharged alive. The change tendencies of total bilirubin level and prothrombin activity were greatly different between patients with in-hospital deaths and patients discharged alive. Some differences in baseline clinical characteristics between different underlying etiologies of pregnant patients with liver failure were also detected.

Conclusions: The mortality rate of liver failure in pregnancy is high especially within 7 days after admission. The change tendencies of total bilirubin and prothrombin activity were greatly different between dead and survived patients with liver failure during hospitalization, which suggested that these parameters might be important prognostic factors of liver failure in pregnancy and their alterations should be carefully tracked.

\section{Background}

Liver failure is featured by hepatic encephalopathy, prolonged prothrombin time and increased levels of aminotransferases, and it is a rare but potentially severe disease with a high rate of short-term morbidity and mortality [1, 2]. Jaundice, coagulation dysfunction, hepatorenal syndrome, hepatic encephalopathy and ascitic fluid are the common clinical manifestations of liver failure in pregnancy [1,2]. Liver failure in pregnancy is defined as the liver failure erupted during pregnancy. The relationship between pregnancy and hepatic function is complex. Pregnancy induces certain physiological changes in the liver, which, if exaggerated, may result in pregnancy-associated acute liver disease [3]. Liver failure in pregnancy is more complicated and more prone to causing complications such as coagulation dysfunction, disseminated intravascular coagulation, postpartum hemorrhage, hepatic encephalopathy, multiple organ dysfunction 
syndrome $[1,4,5]$, and it is one of the main causes of maternal death with a mortality rate reported to be $29 \%-80 \%[6,7]$.

To our best knowledge, due to its low incidence rate, diverse underlying etiologies and complex clinical manifestations, there is still a lack of accurate diagnosis, effective treatments and prognostic indicators for liver failure in pregnancy. Therefore, it is significant to understand the clinical characteristics of liver failure in pregnancy as well as its effective treatments and potential prognostic indicators, in order to promote precise management and improve survival. In China, liver failure in pregnancy is generally divided into two categories: pregnancy-related and non-pregnancy-related liver failure. Pregnancy-related liver failure is mainly caused by acute fatty liver of pregnancy (AFLP); while non-pregnancy-related liver failure is mainly caused by viral hepatitis particularly hepatitis $B[8,9]$. This study aims to retrospectively investigate the clinical characteristics of liver failure in pregnancy caused by AFLP and hepatitis $B$, and to explore the potential prognostic indicators, in order to facilitate the management of liver failure in pregnancy.

\section{Methods}

\section{Study population}

Sixty-two pregnant women with symptoms and signs of hepatic dysfunction, admitting to the Third Affiliated Hospital of Sun Yat-sen University between January 1, 2010 and December 31, 2019 were enrolled in this study. The inclusion criteria were as follows: (1) patients $\geq 18$ years old, with a gestational age of 24-42 weeks and a hospitalization time $\geq 24$ hours; (2) patients with a diagnosis of liver failure confirmed according to the Chinese Guidelines for Diagnosis and Treatment of Liver Failure (2012)[10]; (3) the underlying etiology of liver failure were caused by AFLP or hepatitis B. The exclusion criteria were: (1) the underlying etiology of liver failure were not caused by AFLP or hepatitis B; (2) patients with existing severe heart, brain, or lung diseases, primary kidney disease, or renal dysfunction. All the experiments were approved the Research Ethics Committee of the Third Affiliated Hospital of Sun Yat-sen University, and each patient signed the written informed consent. The study protocol was performed in accordance with the relevant guidelines.

\section{Data collection}

The demographic and baseline clinical data of all patients were collected, including age, gestational age, underlying etiology of liver failure, symptoms, laboratory examinations and therapies during hospitalization. The laboratory examinations included blood routine, coagulation tests, liver function tests, electrolyte, blood glucose and renal function tests. The data of laboratory examinations during hospitalization were collected using last observation carried forward (LOCF) approach [11]. The course of their pregnancy was closely followed and the end point of observation was the discharge or death of the woman.

\section{Statistical analysis}


The continuous variables were presented by median and interquartile range and Wilcoxon rank sum test was used for comparison between groups. Categorical variables were presented by frequency and percentage, and Chi-square test was used for comparison between groups. Cumulative in-hospital death curve was plotted using Kaplan-Meier method. Change tendencies of important laboratory examinations aspartate aminotransferase, total bilirubin, cholinesterase and prothrombin activity were plotted with median values of the first 15 days during hospitalization. Statistical analyses were all performed using IBM SPSS (version 25.0). A two-tailed $\mathrm{P}<0.05$ was considered statistically significant.

\section{Results}

\section{Clinical characteristics of pregnant patients with liver failure}

A total of 62 pregnant patients with liver failure was included in this study. Among these patients, 17 $(17 / 62,27.4 \%)$ patients died during hospitalization. A rapid surge of in-hospital death in the first 7 days after admission was observed (Fig. 1), and the in-hospital mortality rate reached 16.1\% (10 in 62 patients) at day 7 after admission. The increase of mortality rate subsequently slowed down, with a mortality rate of $17.7 \%$ (11 in 62 patients) at day 14, 22.5\% (14 in 62 patients) at day 21, and 27.4\% (17 in 62 patients) at day 30 after admission, and no in-hospital deaths occurred after 30 days.

Table 1 compared the baseline clinical characteristics between patients with in-hospital death and alive. Patients who died in the hospital had a significantly higher proportion of hepatitis $B$ as underlying etiology $(76.5 \%$ vs $40.0 \%, P=0.010)$, lower gestational age at admission ( 242.0 vs 252.0 days, $P=0.043)$, and were more likely to have jaundice $(88.2 \%$ vs $55.3 \%, P=0.011)$ when compared to patients discharged alive (Table 1). No significant difference was found in terms of age, gravidity, parity, pattern of terminating pregnancy and fetal sex between patients died in the hospital and discharged alive (Table 1). 
Table 1

Comparison of baseline clinical characteristics between patients died in the hospital and discharged alive.

\begin{tabular}{|c|c|c|c|}
\hline Variables* & Dead $(n=17)$ & Alive $(n=45)$ & $P$ value \\
\hline Age (years old) & $28.0(24.5-33.5)$ & $28.0(24.5-33.5)$ & 0.806 \\
\hline Etiology & & & 0.010 \\
\hline Hepatitis B & $13(76.5 \%)$ & $18(40.0 \%)$ & \\
\hline Acute fatty liver of pregnancy & $4(23.5 \%)$ & $27(60.0 \%)$ & \\
\hline Hospital stays (days) & $7.0(6.0-17.0)$ & $21.0(17.5-30.0)$ & $<0.001$ \\
\hline Time from diagnosed to delivery (hrs) & $12.0(5.5-42.0)$ & $4.0(2.0-12.0)$ & 0.076 \\
\hline Gestational age (days) & $242.0(179.0-250.0)$ & $252.0(229.5-264.0)$ & 0.043 \\
\hline Gravidity less than three times & $13(76.5 \%)$ & $37(82.2 \%)$ & 0.721 \\
\hline Parity more than twice & $7(41.2 \%)$ & $24(53.5 \%)$ & 0.393 \\
\hline Pattern of terminating pregnancy & & & 0.954 \\
\hline Cesarean delivery & $12(75.0 \%)$ & $32(71.1 \%)$ & \\
\hline Vaginal delivery & $3(18.8 \%)$ & $10(22.2 \%)$ & \\
\hline Forceps & $1(6.3 \%)$ & $3(6.7 \%)$ & \\
\hline Fetal sex & & & 1.000 \\
\hline Boy & $11(73.3 \%)$ & $29(76.3 \%)$ & \\
\hline Girl & $4(26.7 \%)$ & $9(23.7 \%)$ & \\
\hline \multicolumn{4}{|l|}{ Symptoms } \\
\hline Jaundice & $15(88.2 \%)$ & $24(53.3 \%)$ & 0.011 \\
\hline Diarrhea or nausea & $8(47.1 \%)$ & $25(55.6 \%)$ & 0.550 \\
\hline Fatigue & $5(29.4 \%)$ & $9(20.0 \%)$ & 0.502 \\
\hline \multicolumn{4}{|l|}{ Complications } \\
\hline Hepatic encephalopathy & $8(47.1 \%)$ & $10(22.2 \%)$ & 0.067 \\
\hline Disseminated intravascular coagulation & $5(29.4 \%)$ & $4(8.9 \%)$ & 0.099 \\
\hline Multiple organ dysfunction syndrome & $2(11.8 \%)$ & $3(6.7 \%)$ & 0.609 \\
\hline Hepatorenal syndrome & $3(17.6 \%)$ & $0(0.0 \%)$ & 0.018 \\
\hline Postpartum hemorrhage & $9(52.9 \%)$ & $17(37.7 \%)$ & 0.311 \\
\hline
\end{tabular}




\begin{tabular}{|llll|}
\hline Variables* & Dead $(\mathbf{n}=\mathbf{1 7})$ & Alive $(\mathbf{n}=\mathbf{4 5})$ & P value \\
\hline Postpartum hemorrhage $(\mathrm{ml})$ & $1425(375-1500)$ & $500(305-1300)$ & 0.143 \\
\hline Treatments & & & \\
\hline Hysterectomy & $9(52.9 \%)$ & $9(20.0 \%)$ & 0.025 \\
\hline Intrauterine balloon tamponade & $5(29.4 \%)$ & $27(60.0 \%)$ & 0.032 \\
\hline Plasmapheresis & $7(41.2 \%)$ & $27(60.0 \%)$ & 0.184 \\
\hline Continuous renal replacement therapy & $4,23.5 \%$ & $21(46.7 \%)$ & 0.098 \\
\hline
\end{tabular}

*Variables were presented as $\mathrm{n}(\%)$ or median (interquartile range) as appropriate. During hospitalization, 18 of $62(29.0 \%)$ pregnant patients with liver failure developed hepatic encephalopathy (Table 1). Patients died in the hospital had a numerically higher incidence rate of hepatic encephalopathy (47.1\% vs $22.2 \%)$, while the difference was not statistically significant $(P=0.067$; Table 1). There were no significant differences in incidence rates of DIC $(29.4 \%$ vs $8.9 \%, P=0.099)$ and postpartum hemorrhage $(52.9 \%$ vs $37.7 \%, \mathrm{P}=0.311)$ between patients discharged alive and dead (Table 1). The incidence rate of hepatorenal syndrome was significantly higher in patients died in the hospital than that in patients discharged alive (17.6\% vs $0.0 \%, P=0.018$; Table 1$)$.

In terms of the treatments received by the patients, significantly higher proportion of died patients received hysterectomy $(52.9 \%$ vs $20.0 \%, \mathrm{P}=0.025)$ and lower proportion of them received intrauterine balloon tamponade ( $29.4 \%$ vs $60.0 \%, \mathrm{P}=0.032$ ) when compared to patients discharged alive (Table 1 ).

\section{Comparison of laboratory examination parameters of pregnancy patients died in the hospital or discharged alive}

As for baseline laboratory examinations, patients died in the hospital had significantly higher aspartate aminotransferase (492.0 vs $200.0 \mathrm{U} / \mathrm{L}, \mathrm{P}=0.026$ ), total bilirubin (274.6 vs $206.9 \mu \mathrm{mol} / \mathrm{L}, \mathrm{P}<0.001$ ), direct bilirubin (175.4 vs $155.8 \mu \mathrm{mol} / \mathrm{L}, \mathrm{P}=0.007$ ), indirect bilirubin (103.4 vs $54.6 \mu \mathrm{mol} / \mathrm{L}, P<0.001$ ), and platelet (185.0 vs $\left.150.0 \times 10^{9} / \mathrm{L}, \mathrm{P}=0.001\right)$, and lower cholinesterase (2720.0 vs $\left.3462.0 \mathrm{U} / \mathrm{L}, \mathrm{P}<0.001\right)$, creatinine (53.0 vs $141.0 \mu \mathrm{mol} / \mathrm{L}, \mathrm{P}=0.001)$, and PTA ( $21.55 \%$ vs $36.25 \%, P=0.020$ ) when compared to patients discharged alive (Table 2). No significant difference was found in other laboratory examination parameters between patients died in the hospital and discharged alive (Table 2). 
Table 2

Comparison of laboratory examination parameters between pregnant patients died in the hospital and discharged alive.

\begin{tabular}{|llll|}
\hline Variables* & Dead $(\mathbf{n}=17)$ & Alive $(\mathbf{n}=45)$ & P value \\
\hline Aspartate aminotransferase $(\mathrm{U} / \mathrm{L})$ & $492.0(166.0-997.5)$ & $200.0(115.5-489.0)$ & 0.026 \\
\hline Alanine aminotransferase $(\mathrm{U} / \mathrm{L})$ & $361.0(285.0-576.5)$ & $233.0(112.5-450.0)$ & 0.080 \\
\hline Albumin $(\mathrm{g} / \mathrm{L})$ & $26.9(22.6-28.5)$ & $27.3(26.4-29.3)$ & 0.160 \\
\hline Total bilirubin $(\mu \mathrm{mol} / \mathrm{L})$ & $274.6(219.5-371.7)$ & $206.9(155.6-234.7)$ & 0.001 \\
\hline Direct bilirubin $(\mu \mathrm{mol} / \mathrm{L})$ & $175.4(158.6-231.7)$ & $155.8(119.8-175.2)$ & 0.007 \\
\hline Indirect bilirubin $(\mu \mathrm{mol} / \mathrm{L})$ & $103.4(66.4-137.5)$ & $54.6(29.5-71.6)$ & $<0.001$ \\
\hline Cholinesterase $(\mathrm{U} / \mathrm{L})$ & $2720.0(2341.5-3220.5)$ & $3462.0(3168.5-4074.5)$ & $<0.001$ \\
\hline Blood glucose $(\mathrm{mmol} / \mathrm{L})$ & $4.44(3.32-5.40)$ & $4.20(3.32-5.00)$ & 0.523 \\
\hline Total bile acid $(\mu \mathrm{mol} / \mathrm{L})$ & $117.2(87.6-199.9)$ & $102.0(75.3-135.1)$ & 0.316 \\
\hline Blood urea nitrogen $(\mathrm{mmol} / \mathrm{L})$ & $3.04(1.29-5.64)$ & $5.58(2.64-7.01)$ & 0.084 \\
\hline Creatinine $(\mu \mathrm{mol} / \mathrm{L})$ & $53.0(44.5-126.2)$ & $141.0(71.0-190.5)$ & 0.001 \\
\hline Potassium $(\mathrm{mmol} / \mathrm{L})$ & $3.66(3.19-4.13)$ & $3.87(3.61-4.29)$ & 0.055 \\
\hline Sodium $(\mathrm{mmol} / \mathrm{L})$ & $137.4(134.1-139.5)$ & $134.3(132.0-139.0)$ & 0.174 \\
\hline White blood cell $\left(\times 10^{9} / \mathrm{L}\right)$ & $13.29(11.12-14.86)$ & $14.12(12.05-16.99)$ & 0.240 \\
\hline Neutrophil percentage & $0.76(0.72-0.82)$ & $0.76(0.72-0.82)$ & 0.862 \\
\hline Hemoglobin $(\mathrm{g} / \mathrm{L})$ & $110.6(100.0-114.0)$ & $110.6(97.5-129.5)$ & 0.364 \\
\hline Hematocrit & $0.30(0.29-0.32)$ & $0.32(0.28-0.37)$ & 0.131 \\
\hline Platelet $\left(\times 10^{9} / \mathrm{L}\right)$ & $185.0(167.1-272.0)$ & $150.0(119.5-181.0)$ & 0.001 \\
\hline Prothrombin activity $(\%)$ & $21.55(15.25-36.25)$ & $34.00(25.00-40.00)$ & 0.020 \\
\hline Fibrinogen $(\mathrm{g} / \mathrm{L})$ & $1.12(0.61-1.63)$ & $1.20(0.61-1.52)$ & 0.734 \\
\hline
\end{tabular}

*Variables were presented as median (interquartile range) as appropriate.

The aspartate aminotransferase levels of patients from both groups peaked at admission, decreased rapidly to $<100 \mathrm{U} / \mathrm{L}$ within 1-2 days after admission, and tended to be stable and returned to normal range thereafter (Fig. 2A). The total bilirubin levels of patients from both groups decreased in the first 3 days after admission, but after that, total bilirubin level in patients died in the hospital started to rise, while total bilirubin level in patients discharged alive continued to decrease (Fig. 2B). The cholinesterase level in patients from both patients reached the nadir at admission and then gradually increased (Fig. 2C). Patients from both groups had a severe decrease in prothrombin activity at admission. After 
admission, prothrombin activity in patients discharged alive recovered gradually and approached normal range at day 15 after admission, while that in patients died in the hospital showed no tendency of recovery (Fig. 2D).

\section{Discussion}

The present study found that the in-hospital mortality rate of liver failure in pregnancy was $27.4 \%$ and most of the deaths were recorded in the first 7 days after admission. Patients suffered in-hospital death had a significant lower gestational age, a higher incidence rate of hepatorenal syndrome and were more likely to receive hysterectomy but less likely to receive intrauterine balloon tamponade. The baseline aspartate aminotransferase, total bilirubin, indirect bilirubin and platelet levels were significantly higher, while cholinesterase, prothrombin activity and creatinine levels were significantly lower in patients who died in the hospital than that in patients discharged alive. The change tendencies of total bilirubin level and prothrombin activity were greatly different between patients died in the hospital and patients discharged alive. Moreover, patients with liver failure caused by hepatitis $B$ had a higher in-hospital mortality rate compared with those caused by AFLP.

The incidence rate of liver failure in pregnancy is about 1-10 per million person-years but with a high mortality rate, and it is one of the main indirect factors leading to maternal death [12]. In this study, the inhospital mortality rate of patients with liver failure was $27.4 \%$, which was similar to that previously reported in China [13]. From the cumulative death curve, we found that the mortality rate of patients with liver failure increased rapidly within 7 days after admission, while it slowed down after 7 days. As delivery were carried out as soon as possible in patients with liver failure, the results indicated that delivery was an important factor leading to the rapid change of the condition in patients with liver failure. Liver failure in pregnancy might develop rapidly after delivery and the risk of death becomes highest in a short period of 7 days. Thus, in clinical practice, it is necessary to carefully and actively manage patients with liver failure in the early stage to reduce mortality. It was reported that patients with liver failure was mainly of late pregnancy (after 28 weeks), which was consistent with what was found in the present study. Besides, the gestational age of patients died in the hospital was significantly lower, suggesting that the early onset of liver failure in pregnancy might be related to poor prognosis. The results also found that the postpartum bleeding rate was numerically higher in those died during hospitalization, and the hysterectomy rate was significantly higher in patients died in the hospital. On the contrary, the utilization rate of intrauterine balloon tamponade was significantly higher in those survived. Patients with liver failure frequently suffered from coagulation dysfunction, leading to a higher risk of postpartum hemorrhage than normal pregnant women. Postpartum hemorrhage could further aggravate coagulation dysfunction and lead to a vicious circle. Vrachnis et al. reported that the success rate of hemostasis was $94.4 \%$ in postpartum hemorrhage patients treated with intrauterine balloon tamponade [14]. However, there has been no study in the application of intrauterine balloon tamponade in patients with liver failure. In this study, 32 patients were treated with intrauterine balloon tamponade and 27 of them survived, suggesting that active use of intrauterine balloon tamponade in patients with liver failure might alleviate postpartum hemorrhage and improve survival rate. 
The change tendencies of important laboratory examinations in patients with liver failure illustrated that total bilirubin and prothrombin activity changed in totally different ways in patients died in the hospital or discharged alive, while changes in aspartate aminotransferase and cholinesterase changes were similar between the two groups. Of note, though having a similar change tendency, aspartate aminotransferase was significantly higher at baseline in patients suffered in-hospital death. Aspartate aminotransferase is an intracellular functional enzyme of the liver. After the damage and destruction of hepatocytes, aspartate aminotransferase will soon be released into the blood, therefore the level of aspartate aminotransferase can sensitively reflect the degree of necrosis of hepatocytes especially in the onset stage of the disease $[15,16]$. However, when the disease progresses, the level of aspartate aminotransferase will tend to be stable or even decreased with a broad necrosis of hepatocytes, but the increase of total bilirubin will persist. When liver disease progresses to liver failure, considerable amounts of hepatocytes have been damaged, which is in line with the results of this study that the aspartate aminotransferase level reached the peak at admission. As the disease progressively deteriorated in patients who finally dead during hospitalization, the aspartate aminotransferase level of them decreased, while the total bilirubin level continuously increased, leading to the phenomenon "bilirubin-transaminase separation", which could indicate poor prognosis [13]. In survived patients, the levels of aspartate aminotransferase and total bilirubin decreased simultaneously, indicating that the injury of hepatocytes might have been slowed down or even stopped and the liver gradually recovered. It has been reported that cholinesterase level reflected the synthesis function of the liver and was related to the prognosis of patients with liver failure $[17,18]$. In the present study, the level of cholinesterase reached the nadir at admission and increased after that. Within 7 days after admission, the cholinesterase level of in-hospital dead patients was lower than those survived, suggesting that the liver damage was more severe among in-hospital dead patients. However, the change tendencies didn't differ significantly among discharge dead or alive patients, indicating that the baseline cholinesterase level might be more closely associated with prognosis. Most of the coagulation factors are synthesized in the liver, thus coagulation dysregulation is one of the parameters reflecting liver dysfunction. The important coagulation function marker, prothrombin activity, has been reported to be related tightly to prognosis in liver failure patients [19-21]. In the present study, prothrombin activity decreased apparently at baseline in patients with liver failure. But after admission, an increasing trend was observed in patients survived while no improvement was found in died patients. These results indicated that liver synthesis function was poor and coagulation dysfunction was difficult to be corrected in in-hospital dead patients with liver failure, and that the dynamic change of prothrombin activity level was associated with the prognosis of patients with liver failure and should be carefully tracked during hospitalization.

There are some limitations of this study. Firstly, due to the rarity of liver failure in pregnancy, the sample size of this study is still relatively small. Secondly, this is a single-center study, which may affect the generalizability of the results. Thirdly, as a retrospective analysis, bias caused by data collection and management amendment might exist.

\section{Conclusions}


To summarize, the mortality rate of liver failure in pregnancy is high especially within 7 days after admission. In clinical practice, we should pay more attention to and take timely actions on pregnant patients with liver failure especially in the first 7 days after admission. The change tendencies of total bilirubin and prothrombin activity were greatly different between dead and survived patients with liver failure during hospitalization, which suggested that these parameters might be important prognostic factors of liver failure in pregnancy and their alterations should be carefully tracked.

\section{Declarations}

\section{Ethics approval and consent to participate}

All the experiments were approved the Research Ethics Committee of the Third Affiliated Hospital of Sun Yat-sen University, and each patient signed the written informed consent. The study protocol was performed in accordance with the relevant guidelines.

\section{Consent for publication}

All the authors approved the submission of the manuscript.

\section{Availability of data and materials}

All the data are available upon reasonable request from the corresponding author.

\section{Competing interests}

None.

\section{Funding}

None.

\section{Authors' contributions}

$\mathrm{ZH}, \mathrm{YY}$ and $\mathrm{YZ}$ conceptualized the study and was involved in study design; $\mathrm{ZH}$ and JZ collected the data and performed the follow-up; $\mathrm{PZ}, \mathrm{ZT}$ and TH performed the data analysis; $\mathrm{YY}$ and $\mathrm{YZ}$ drafted the manuscript and $\mathrm{HH}$ revised it critically. All the authors approved for the manuscript submission.

\section{Acknowledgements}

None.

\section{References}

1. Bacak SJ, Thornburg LL: Liver Failure in Pregnancy. Critical care clinics 2016, 32(1):61-72. 
2. Liu J, Ghaziani TT, Wolf JL: Acute Fatty Liver Disease of Pregnancy: Updates in Pathogenesis, Diagnosis, and Management. The American journal of gastroenterology 2017, 112(6):838-846.

3. Bernal W, Wendon J: Acute liver failure. The New England journal of medicine 2013, 369(26):25252534.

4. Hay JE: Liver disease in pregnancy. Hepatology (Baltimore, Md) 2008, 47(3):1067-1076.

5. Rovegno M, Vera M, Ruiz A, Benítez C: Current concepts in acute liver failure. Annals of hepatology 2019, 18(4):543-552.

6. Riordan SM, Williams R: Perspectives on liver failure: past and future. Seminars in liver disease 2008, 28(2):137-141.

7. Bernal W, Hyyrylainen A, Gera A, Audimoolam VK, McPhail MJ, Auzinger G, Rela M, Heaton N, O'Grady JG, Wendon $\mathrm{J}$ et al: Lessons from look-back in acute liver failure? A single centre experience of 3300 patients. Journal of hepatology 2013, 59(1):74-80.

8. Chen H, Yuan L, Tan J, Liu Y, Zhang J: Severe liver disease in pregnancy. International journal of gynaecology and obstetrics: the official organ of the International Federation of Gynaecology and Obstetrics 2008, 101(3):277-280.

9. Cheng N, Xiang T, Wu X, Li M, Xie Y, Zhang L: Acute fatty liver of pregnancy: a retrospective study of 32 cases in South China. The journal of maternal-fetal \& neonatal medicine: the official journal of the European Association of Perinatal Medicine, the Federation of Asia and Oceania Perinatal Societies, the International Society of Perinatal Obstet 2014, 27(16):1693-1697.

10. [Guideline for diagnosis and treatment of liver failure]. Zhonghua gan zang bing za zhi = Zhonghua ganzangbing zazhi = Chinese journal of hepatology 2019, 27(1):18-26.

11. Joshi D, James A, Quaglia A, Westbrook RH, Heneghan MA: Liver disease in pregnancy. Lancet (London, England) 2010, 375(9714):594-605.

12. Jashnani KD, Rupani AB, Wani RJ: Maternal mortality: an autopsy audit. Journal of postgraduate medicine 2009, 55(1):12-16.

13. Yang Y, Deng L, Li X, Shi Z, Jiang P, Chen D, Yu Y, Wang Z, Tang X, Zhao S et al: Analysis of prognosis-associated factors in fulminant viral hepatitis during pregnancy in China. International journal of gynaecology and obstetrics: the official organ of the International Federation of Gynaecology and Obstetrics 2011, 114(3):242-245.

14. Vrachnis N, Salakos N, lavazzo C, Grigoriadis C, lliodromiti Z, Siristatidis C, Katsetos C, Creatsas G: Bakri balloon tamponade for the management of postpartum hemorrhage. International journal of gynaecology and obstetrics: the official organ of the International Federation of Gynaecology and Obstetrics 2013, 122(3):265-266.

15. Bitar R, Thwaites R, Davison S, Rajwal S, McClean P: Liver Failure in Early Infancy: Aetiology, Presentation, and Outcome. Journal of pediatric gastroenterology and nutrition 2017, 64(1):70-75.

16. Smith AK, Ropella GEP, McGill MR, Krishnan P, Dutta L, Kennedy RC, Jaeschke H, Hunt CA: Contrasting model mechanisms of alanine aminotransferase (ALT) release from damaged and 
necrotic hepatocytes as an example of general biomarker mechanisms. PLoS computational biology 2020, 16(6):e1007622.

17. Pan C, Xu LJ, Zhou R, Zhou W, Huang JR: [Multivariate analysis of hepatic encephalopathy occurrence in patients with liver failure]. Zhonghua gan zang bing za zhi = Zhonghua ganzangbing zazhi = Chinese journal of hepatology 2012, 20(6):434-437.

18. Tan L, Meng Y, Zeng T, Wang Q, Long T, Wu S, Guan X, Fu H, Zheng W, Tian Y et al: Clinical diagnostic significance of prealbumin, cholinesterase and retinol binding protein in liver cirrhosis combined with encephalopathy. British journal of biomedical science 2019, 76(1):24-28.

19. Khan R, Koppe S: Modern Management of Acute Liver Failure. Gastroenterology clinics of North America 2018, 47(2):313-326.

20. Zhang YP, Kong WQ, Zhou SP, Gong YH, Zhou R: Acute Fatty Liver of Pregnancy: A Retrospective Analysis of 56 Cases. Chinese medical journal 2016, 129(10):1208-1214.

21. Meng J, Wang S, Gu Y, Lv H, Jiang J, Wang X: Prenatal predictors in postpartum recovery for acute fatty liver of pregnancy: experiences at a tertiary referral center. Archives of gynecology and obstetrics 2016, 293(6):1185-1191.

\section{Figures}




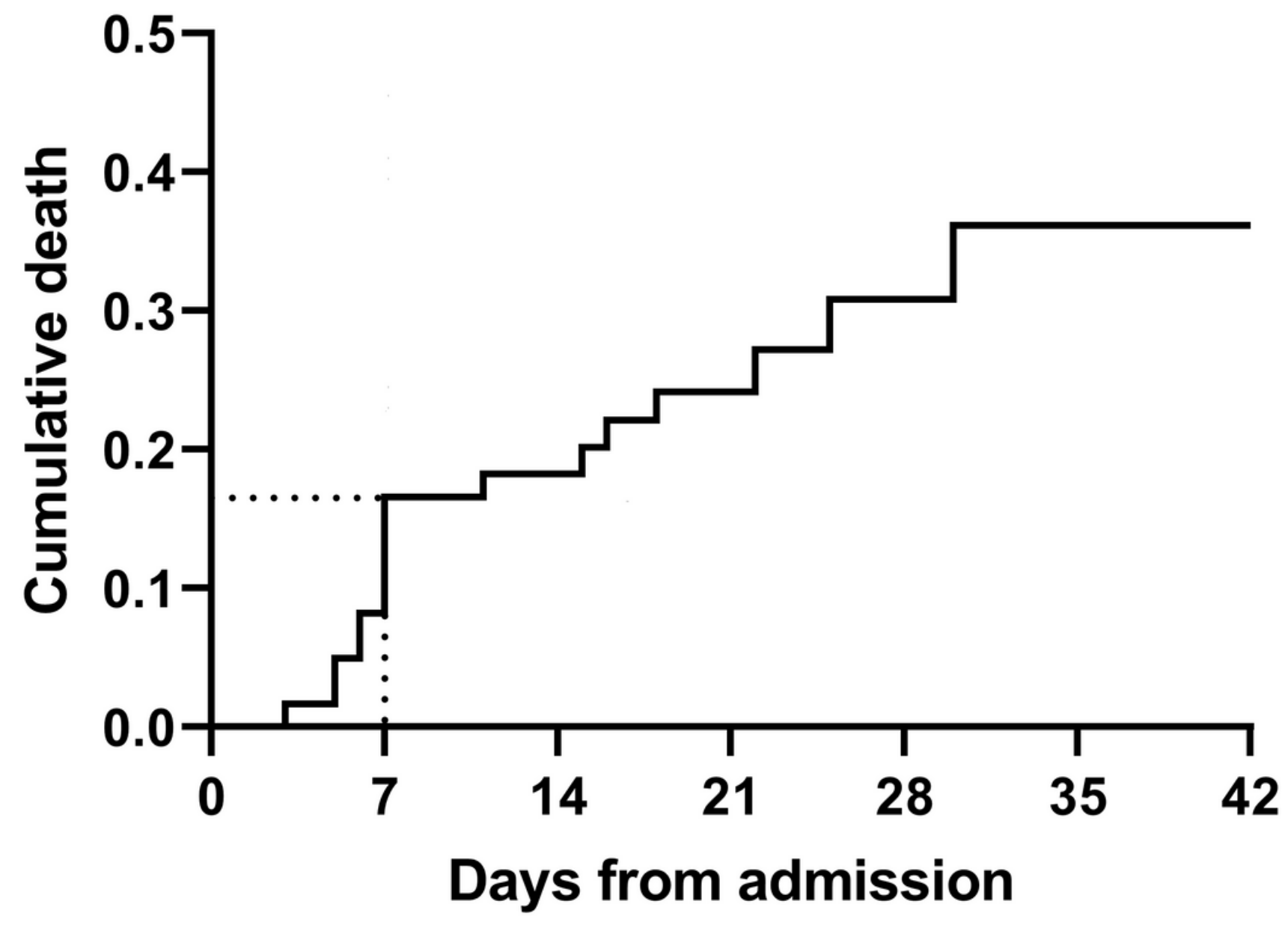

Figure 1

Cumulative in-hospital death curve of pregnant patients with liver failure. 

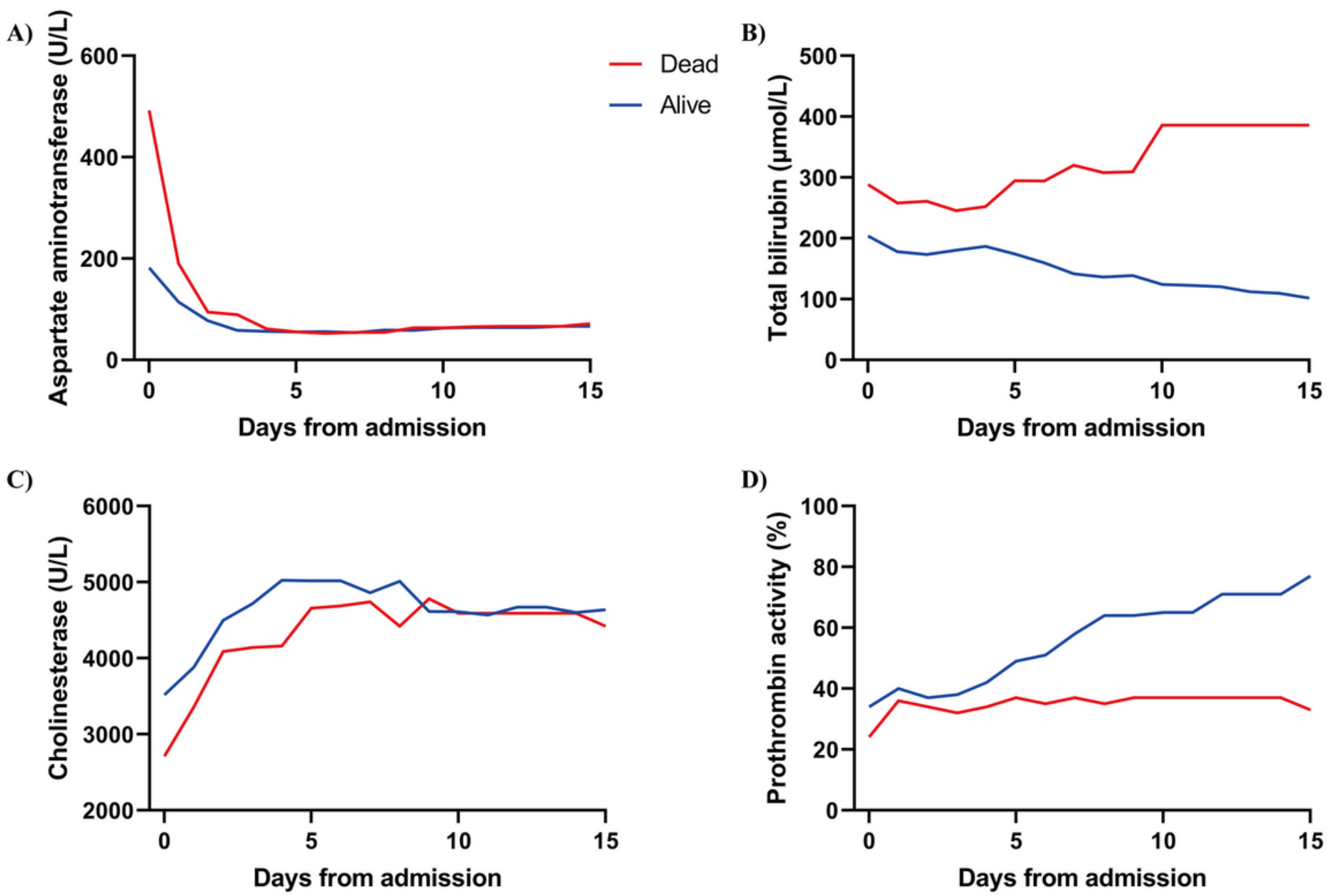

Figure 2

Temporal changes of laboratory examination parameters in pregnant patients with liver failure. Changes of (A) aspartate aminotransferase, (B) total bilirubin, (C) cholinesterase, and (D) prothrombin activity of pregnant patients with liver failure at day 0 to day 15 after admission. 